company. In order to determine the prevalence of stress in a port company in Abidjan we conducted this study.

Method A cross-sectional descriptive and analytical survey was conducted from April 2015 to July 2016. In collaboration with the company's managers, we sensitised staff on the concept of stress at work. The data were collected using three self-questionnaires (the 26-item KARASEK job content questionnaire, 12 items GOLDBERG self-assessment questionnaire for psychopathology, and the French version of the SIEGRIST questionnaire at 46 items).

Results The prevalence of stress was $54.6 \%$. Stress came mainly from supervisors $(65.7 \%)$ and was favoured by work situations such as emergency tasks (21.4\%), poor organisation of work (20.4\%), and Heavy workload (17.8\%). More than two-thirds of stressed workers (64\%) had psychological distress. Age $(p=0.139)$, gender $(p=0.57)$, workplace $(p=0.65)$, professional seniority $(p=0.65)$ and the hourly organisation of work $p=0.39)$ had no statistically significant impact on the occurrence of stress.

Conclusion Stress is a major psychological risk requiring preventive measures such as listening cells and improved working conditions in human, technical and organisational terms.

\section{ASSOCIATION BETWEEN WORKPLACE SOCIAL CAPITAL AND JOB PERFORMANCE: A MULTILEVEL STUDY IN CHINA}

${ }^{1}$ Junling Gao*, ${ }^{2}$ Jing Wang, ${ }^{2}$ Denglai Yu, ${ }^{1}$ Junming Dai, ${ }^{2} \mathrm{Hua}$ Fu, ${ }^{1}$ Yongkai Zhu. ${ }^{1}$ School of Public Health, Fudan University, Shanghai, China; ${ }^{2}$ Shanghai Pudong New Area Centre for Disease Control and Prevention, Shanghai, China

\subsection{6/oemed-2018-ICOHabstracts. 1736}

Introduction Although researchers have recently paid more attention workplace social capital(WSC), which was found to associate with health and health-related behaviours, such as smoking, physical activity and medicine adherence. Theoretically, WSC facilitates to solve collective action problems, so WSC may associate with job performance. However, there was sparse evidence on association between WSC and job performance, and most of them conducted in developed countries. In order to fill the gap, a multilevel study was conducted in shanghai, China.

Methods A cross-sectional study was conducted among 2380 workers from 33 workplace in Shanghai from December 2016 to March 2017. Job performance was measured by The World Health Organisation Health and Work Performance Questionnaire. WSC was measured by Chinese version of Workplace Social Capital Scale. Data were analysed using STATA 13.0.

Results Four-week relative absenteeism was 0.055 (95\% CI: 0.045 to 0.065$)$, and relative presenteeism was $1.037(95 \%$ CI: 1.030 to 1.045$)$. Multilevel generalised linear models indicated that individual-level WSC was negatively associated with relative absenteeism ( $\beta=-0.065,95 \% \mathrm{CI}:-0.100$ to -0.029$)$, both individual-level and aggregated-level WSC were positively associated with relative presenteeism $(\beta=0.038$, 95\% CI: 0.015 to $0.061 ; \beta=0.042,95 \% \mathrm{CI}: 0.019$ to 0.065$)$ after controlling for demographic characteristic and self-rated health.

Conclusion Consistent with previous findings, the current study found that workplace social capital is also associated with job performance in China context. So building workplace social capital may improve job performance.
626

RELATIONSHIP OF QUALITY OF WORK LIFE WITH DEPRESSION/ANXIETY IN ADMINISTRATIVE STAFF OF AN INSTITUTION OF HIGHER EDUCATION IN MEXICO

${ }^{1} \mathrm{R}$ González-Baltazar*, 'SG León-Cortés, ${ }^{1} \mathrm{MI}$ Contreras-Estrada, ${ }^{1} \mathrm{G}$ Hidalgo-Santacruz, ${ }^{2}$ BJ Hidalgo-González. 'Public Health Department, University of Guadalajara, Guadalajara, Mexico; ${ }^{2}$ Gestalt Institute of Guadalajara, Guadalajara, Mexico

10.1136/oemed-2018-ICOHabstracts. 1737

Introduction Currently the Quality of Work Life (QWL) and psychosocial risks are identified as important indicators of health in workers, it is important to carry out studies that can describe the behaviour of them in organisations. The aim was to analyse the relationship between QWL and the presence of symptoms of depression or anxiety in the administrative female employees of a public university in Mexico.

Methods These study was performed with a universe of 6455 employees and a probabilistic sample of 225 people. They all were applied the CVT-GOHISALO and Goldberg's depression and anxiety questionnaires to measure their QWL and the presence of depression or anxiety symptoms. The Cronbach's Alpha of CVT-GOHISALO is 0.9527 and the Goldberg's depression and anxiety scale is 0.89 .

Results Only in one of the seven dimensions of CVT-GOHISALO instrument, the highest percentage of satisfaction was of high level, $49.3 \%$, in all other dimensions the highest percentage of satisfaction was of low level. As for the presence of depression symptoms, $20.9 \%$ of the employees had them and anxiety symptoms occurred in 59.1\%.

In all dimensions, the higher satisfaction with the QWL and the absence of depression or anxiety symptoms were related. As well as low and medium level of satisfaction with the presence of depression or anxiety symptoms. All crosses were statistically significant, with $\mathrm{p}$ values for each dimension ranging between $0.000-0.046$.

Conclusions To the university administrative staff, there is a relationship between being satisfied with the QWL and the absence of depression or anxiety symptoms. High satisfaction with QWL, is a protective factor for not having depression or anxiety symptoms. The risk of developing depression or anxiety symptoms, is four times higher for those with low satisfaction level with their QWL than for those who have high satisfaction level.

\section{COMBINED EFFECTS OF OVERTIME WORK HOURS AND EXERCISE HABITS ON PSYCHOLOGICAL DISTRESS: A CROSS-SECTIONAL STUDY AMONG JAPANESE WHITE- COLLAR WORKERS}

${ }^{1}$ Ayako Hino*, ${ }^{1}$ Yumi Wakida, ${ }^{1}$ Yusuke Noguchi, ${ }^{1}$ Haruka Ido, ${ }^{2}$ Akiomi Inoue, ${ }^{1}$ Kosuke Mafune, ${ }^{1}$ Hisanori Hiro. ${ }^{1}$ University of Occupational and Environmental Health, Japan, Kitakyushu, Japan; ${ }^{2}$ Kitasato University, Sagamihara, Japan

\subsection{6/oemed-2018-ICOHabstracts. 1738}

Introduction As symbolised by the word 'Karoshi' (death from overwork), the effect of overtime work hours on employees' physical and mental health is an important issue in Japan. Although there are inconsistent findings regarding the association between overtime work hours and mental health, several studies have found that physical activity has a positive effect on mental health. In this study, we investigated the combined 